\title{
The Modeling and Equalization Technique of Nonlinear Wireless Channel
}

\author{
Qiu Min*, Mouyannan and Zhang Xiuping \\ Physical Chemistry Department of Heihe University, Heihe 164300, China
}

\begin{abstract}
In this paper, we have considered a new method for modeling and equalizing of nonlinear wireless channel. The Volterra structure equalizer has high computation burden and slow convergence rate due to its large number of coefficients and the pre-distortion method is very difficult to realize in actual wireless communication systems. Here, we have taken another scheme instead of those mentioned above. First, it is assumed that the nonlinear channel under consideration has a Wiener model representation. After fully estimating the parameters of the channel, which consist of a linear filter with memory and a nonlinear filter without memory, we took Hammerstein model equalizer to equalize the channel. The simulation results show the feasibility of the program by applying it to 8PSK wireless communication channel.
\end{abstract}

Keywords: Equalization, Hammerstein model, Nonlinear wireless channel, Wiener model.

\section{INTRODUCTION}

In recent years, the problem about modeling and equalization of digital wireless channel has aroused extensive attention, and a large amount of literature has emerged. Due to limited internal power of the wireless network, it is a huge challenge for scholars to make full use of the limited power to improve the efficiency of digital wireless communication.

Traveling wave tube amplifier (TWTA) in wireless operation in or near the saturation point is the main reason that leads to nonlinear characteristics of the wireless channel [1]. Bringing in Input Back-Off cannot take full advantage of the limited internal power of the wireless network. Therefore, it is generally ensured that the operating point of the amplifier is stabilized at or near the saturation point. Thus, the nonlinear distortion becomes inevitable. It is therefore necessary to establish a reasonable model, and to take appropriate compensation technology to counteract this nonlinearity. The compensation method of nonlinear wireless channel consists of pre-distortion method and nonlinear equalization method, which processes signal at the transmitting and receiving end of the wireless communication system, respectively. The signal pre-distortion method and nonlinear equalization techniques all use mathematical models to represent the inverse characteristics of the channel. However, data predistortion method processes signal constellation directly, which does not need to fit information of the channel.

The literature in modeling and compensating methods for nonlinear wireless channel is mainly about the research of Volterra series, which includes Volterra series model of the nonlinear wireless channel model and its improved method $[2,3]$ and the equalization method for Volterra structure equalizer of nonlinear wireless channel [1]. Contents of these studies

*Address correspondence to this author at the Physical Chemistry Department of Heihe University, Heihe 164300, China; Tel: +13555 202209; E-mail: qm2209@126.com have certain limitations. Moreover, the Volterra structure equalizer has a large number of coefficients and it is not suitable for real-time implementation, so it has been limited in practical applications.

The Wiener model of the wireless channel has been proposed and the channel pre-distorter has been researched, but, in practical applications, pre-distortion method is very difficult to realize. Therefore, generally equalization technology has been researched [5]. In this paper, we have proposed a new equalization scheme based on the Wiener modeling method of wireless channel. We assumed that the considered nonlinear wireless channel can be represented by the Wiener model. The parameters of Wiener model can be estimated [3], and then the Hammerstein model can be used. Simulation results show that the model estimator of the channel and the channel equalizer can obtain convergence. So the proposed scheme is feasible and effective.

\section{WIENER MODEL OF NONLINEAR WIRELESS CHANNEL}

Wiener model is commonly used in identification and modeling for nonlinear systems. It has aroused widespread attention because its structure is simple and it can describe many of the common nonlinear characteristics. Structure of the Wiener model is shown in Fig. (1), where, $w$ indicates the coefficient of the linear part of Wiener model and $f(n)$ indicates the coefficient of the nonlinear part of model. Moreover, the intermediate signal $x(n)$ cannot be measured.

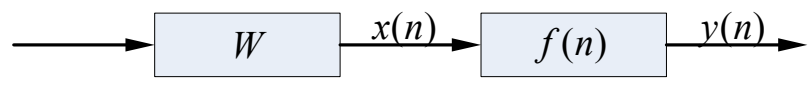

Fig. (1). Structure of Wiener model.

The input-output expressions of the Wiener model are written as:

$$
x(n)=\sum_{i=0}^{N} w_{i} a(n-i)
$$


$y(n)=\sum_{j=1}^{M} f_{j}(x(n))^{j}$

It can be seen that the nonlinear characteristics of the wireless channel to some extent have a certain similarity with the input-output characteristics which are described by Wiener model because the wireless high-power amplifier can be expressed as the cascade of a linear filter with memory and a nonlinear filter without memory.

Next, we need to take an actual channel to estimate parameters of the Wiener model. Herein, we chose the deformable model of TWTA models [6] proposed by Saleh to be the object of research; its input - output characteristics are given by:

$S_{\text {out }}(t)=S_{\text {in }}(t) A(r) e^{j \phi(r)}$

$A(r)=\frac{\alpha_{A} r 0}{1+\beta_{A} c r 0 r^{2}}$

$\phi(r)=\frac{\alpha_{\phi}(r 0 r)^{2}}{1+\beta_{\phi}(r 0 r)^{2}}$

Where, $S_{\text {in }}(t)$ and $S_{\text {out }}(t)$ are the input and output signals through the nonlinear portion; and $A(r)$ and $\phi(r)$ represent the AM-AM and AM-PM effect, respectively.

$r=\sqrt{P(t) / \bar{P}}$

$P(t)$ is the instantaneous power of the input signal, $\bar{P}$ is the average power of the input signal, and $r 0$ represents Input Back-off, where, $\quad \alpha_{A}=2.1587, \beta_{A}=1.1517$, $\alpha_{\phi}=4.0033$, and $\beta_{\phi}=9.1040$. After choosing the parameters of channel, estimated according to the following steps.

Step 1: define the input signal as $a(n)$, and let the signal pass through the actual wireless network; the signal generates amplitude distortion and phase rotation. After adding downlink noise, an output signal $d(n)$ is obtained.

Step 2: select the linear and nonlinear order of the Wiener model and initialize the parameters of the model. Let the signal pass through this model, to obtain the output signal $x(n)$.

Step 3: calculate the error $e(n)=d(n)-x(n)$ and $|e(n)|^{2}$; stochastic gradient descent method is used to estimate the model parameters. $\quad \boldsymbol{f}=\left\{f_{1}, f_{2}, f_{3}, \ldots, f_{M}\right\} \quad$ and $\boldsymbol{w}=\left\{w_{1}, w_{2}, w_{3}, \ldots, w_{N}\right\}$ denote linear and nonlinear coefficients of the Wiener model, respectively.

Iterative formula of linear part and nonlinear part is as follows:

$\boldsymbol{w}(n+1)=\boldsymbol{w}(n)+\mu e(n) \Delta_{1}^{*}(n)$

$$
\boldsymbol{f}(n+1)=\boldsymbol{f}(n)+\mu e(n) \Delta_{2}^{*}(n)
$$

Where, ${ }^{*}$ is the conjugate and $\Delta_{1}(n)$ is the corresponding gradient parameter for the estimation of linear parameters; the process is therefore as follows:

$$
\Delta_{1}^{*}(n)=\frac{d(y(n))}{d(\boldsymbol{w})}=\left\{\frac{\partial(y(n))}{\partial w_{1}}, \frac{\partial(y(n))}{\partial w_{2}}, \ldots, \frac{\partial(y(n))}{\partial w_{N}}\right\}^{*}
$$

Where,

$$
\begin{aligned}
& \frac{\partial(y(n))}{\partial w_{i}}=f_{1} x(n-i)+2 f_{2} x(n) x(n-i)+\ldots+M f_{M} x^{M-1}(n) x \\
& (n-i) \quad(i=0,1, \ldots, N)
\end{aligned}
$$

Where, $\Delta_{2}(n)$ is the corresponding gradient parameter for the estimation of nonlinear parameters; the process is as follows:

$$
\Delta_{2}^{*}(n)=\frac{d(y(n))}{d \boldsymbol{f}}=\left\{x(n), x^{2}(n), x^{3}(n), \ldots, x^{M}(n)\right\}^{*}
$$

From Eq.(6)-Eq.(10), the coefficients can be acquired which belong to linear and nonlinear part of the Wiener model respectively.

\section{HAMMERSTEIN MODEL EQUALIZER OF NON- LINEAR WIRELESS CHANNEL}

Hammerstein model, whose structure is the inverse model of the Wiener model, is shown in Fig. (2).

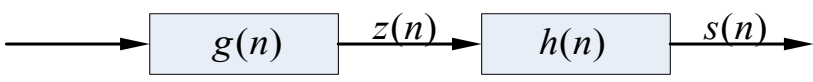

Fig. (2). Structure of Hammerstein model.

In Fig. (2), $g(n)$ represents coefficients of the nonlinear part, whereas $h(n)$ represents coefficients of the linear part. The input-output expressions of the Hammerstein model are written as Eq.(11) and Eq.(12). Linear and nonlinear orders of the Hammerstein model must be greater than the corresponding linear and nonlinear order of the Wiener model.

$$
\begin{array}{ll}
z(n)=\sum_{k=1}^{K} g_{k}(\mathrm{y}(n))^{k} & (K>M) \\
s(n)=\sum_{l=0}^{L} h_{l} z(n-l) & (L>N)
\end{array}
$$

In the areas of modeling and identification of nonlinear systems, the Hammerstein model and the Wiener equalizer of the nonlinear systems are studied [5, 7]. But as we know, Wiener model and Hammerstein model are mutually inverse to each other, that is to say, if we take Wiener model to establish the model of the system, then the Hammerstein model can be taken to establish the model of the equalizer. According to literature [4] and the mutual inverse characteristic of these two models, the Hammerstein model can be placed behind the Wiener model to be the adaptive equalizer of the 
wireless channel. The simulation results have also proven this point.

Assume that $\boldsymbol{g}=\left\{g_{1}, g_{2}, g_{3}, \ldots, g_{K}\right\}$, and $\boldsymbol{h}=\left\{h_{0}\right.$, $\left.h_{1}, h_{2}, \ldots, h_{L}\right\}$ are the coefficients of nonlinear and linear part of the Hammerstein model. The error is expressed as $c(n)=a(\mathrm{n})-s(\mathrm{n})$.

The iterative processes of coefficients of nonlinear and linear part are given by:

$\boldsymbol{g}(n+1)=\boldsymbol{g}(n)+v c(n) \Delta_{1}^{*}(n)$

$\boldsymbol{h}(n+1)=\boldsymbol{h}(n)+v c(n) \Delta_{2}^{*}(n)$

The gradient of nonlinear part is estimated as follows:

$\Delta_{1}^{*}(n)=\left\{\frac{\partial(s(n))}{\partial g_{1}}, \frac{\partial(s(n))}{\partial g_{2}}, \ldots, \frac{\partial(s(n))}{\partial g_{K}}\right\}^{*}$

Where,

$$
\frac{\partial(s(n))}{\partial g_{k}}=h_{0} \mathrm{y}^{k}(n)+h_{1} \mathrm{y}^{k}(n-1)+\ldots+h_{L} \mathrm{y}^{k}(n-L)(k=1,2, \ldots, K)
$$

The gradient of linear part is estimated as follows:

$\Delta_{2}^{*}(n)=\frac{d(s(n))}{d(\boldsymbol{h})}=\{z(n), z(n-1), \ldots, z(n-L)\}^{*}$

From Eq.(13)-Eq.(17), we can get the coefficients which belong to Hammerstein model.

\section{THE SIMULATION RESULTS}

In the process of simulation, we choose $8 P S K$ with $3000(? \mathrm{~m} / \mathrm{cm})$ length. High power amplifier (HPA) of the wireless is regarded as the cascade of FIR filter with memory and nonlinear model without memory. The parameters of linear filter are $\left[\begin{array}{lll}0.7962 & 0.1538 & 0.0769\end{array}\right]$. The Input Back-Off $I B O=8$. Linear and nonlinear orders of the Wiener model are both 3, while linear and nonlinear orders of the Hammer- stein model are both 4 . The step-size of linear and nonlinear part is 0.08 and 0.06 , respectively. The step-size of the equalizer is 0.01 . Center tap method can be taken to initialize the parameters of the Wiener model, while linear and nonlinear taps of the equalizer are both initialized as $[1,0,0,0]$. Iterative curve of parameters estimation for Wiener model is shown in Fig. (3). From Fig. (3), it can be seen that the mean square error converges after about 200 times of iteration. A set of parameters are obtained after convergence. Several tests can be carried out to obtain the optimal value of the model parameters and choose a set of data from 100 independent tests to select the final parameters.

Linear part:

$\mathbf{w}=\left[\begin{array}{lll}1.0451+0.6332 i & -0.0266+0.1361 i & 0.0371+0.0639 i\end{array}\right]$

Nonlinear part:

$\mathbf{g}=\left[\begin{array}{lll}1.2211-0.5451 i & 0.0014+0.0108 i & 0.0016-0.0054 i\end{array}\right]$

In this way, modeling of nonlinear wireless channel with Wiener model can be completed. After obtaining parameters of the Wiener model, the channel is equalized with the Hammerstein equalizer. Iterative curve of parameters estimation for Hammerstein equalizer is shown in Fig. (4). It can be seen that the iterative curve of the Hammerstein equalizer can achieve its convergence after about 300 iterations and converges to about $-100 \mathrm{db}$.

Fig. (5) shows that the receiving signal can generate amplitude distortion and phase rotator, but it can be clearly seen from Fig. (6) that the equalized signal constellations are very clear and the phase rotation has been corrected. It is worth noting that the $I B O$ that was taken into account is relatively a high value compared to the practical ones, which means that the nonlinear distortion is not very serious in the wireless system as considered. In actual situations, the equalization result is always worse than the result here, that is to say, the algorithms and methods based on Hammerstein equalizer are also worth researching.

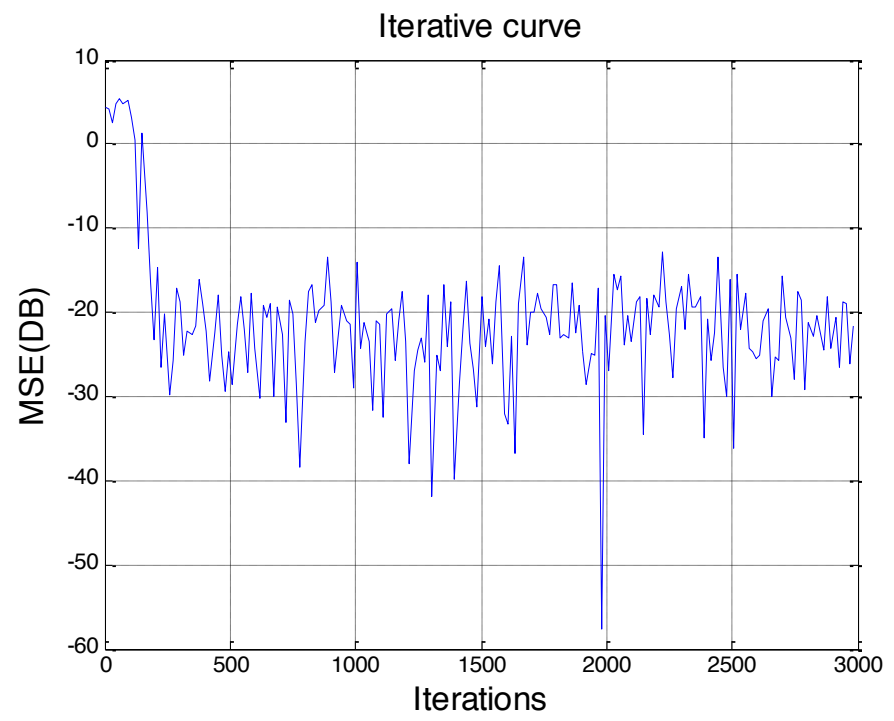

Fig. (3). Parameters estimation of Wiener model. 


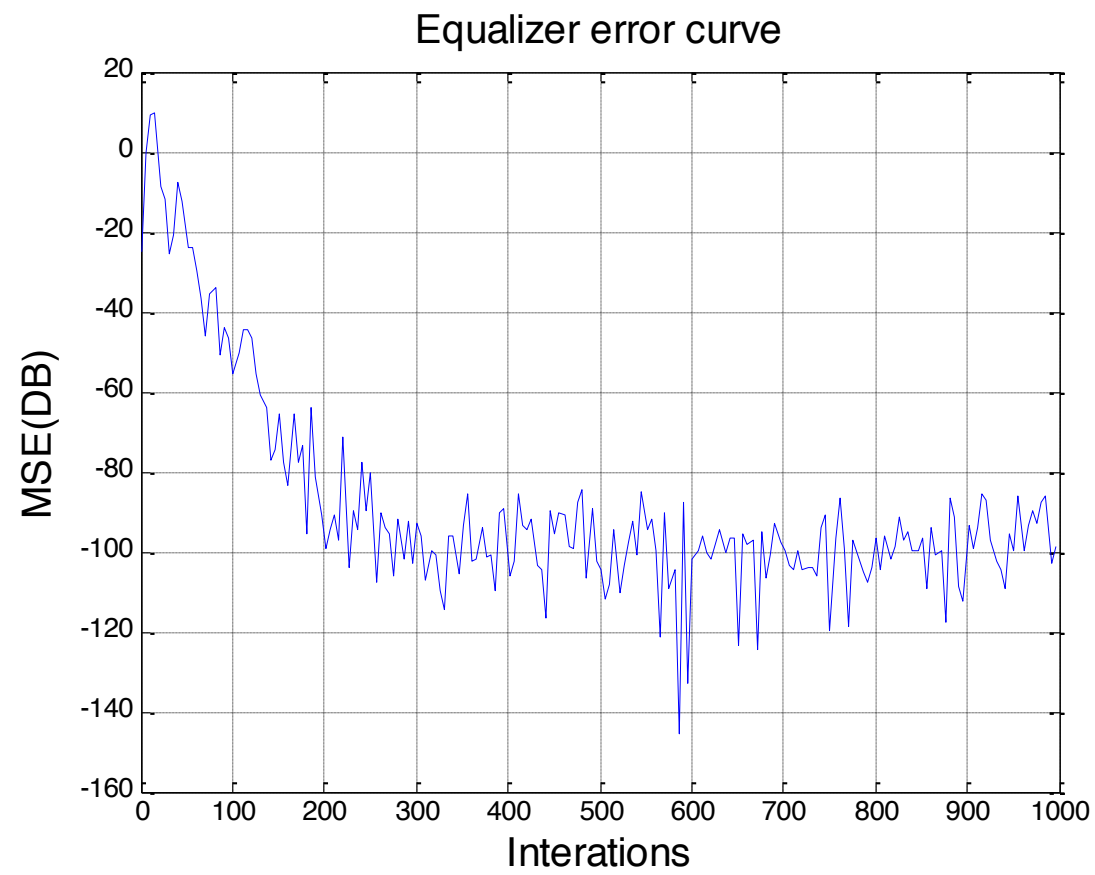

Fig. (4). Parameters estimation of Hammerstein equalizer.

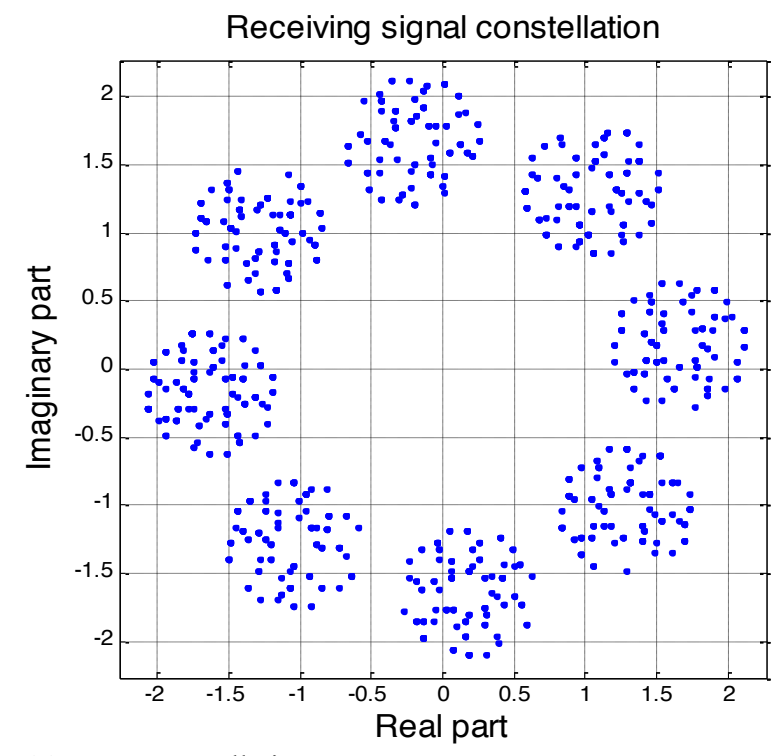

Fig. (5). Input constellations.

\section{CONCLUSION}

In this paper, on the basis of studying the Wiener model of the nonlinear wireless channel, we proposed the idea of using the Hammerstein model as a channel equalizer, and simulation was carried out to prove this method. It is different with the traditional Volterra equalizer. The simulation results show that the Hammerstein equalizer can equalize the nonlinear wireless channel well. Moreover, the algorithms and methods can be further studied based on the Hammerstein equalizer. However, the iterative curve of parameter estimation for the Wiener model still has some fluctuations after convergence, so there is considerable deviation between the model and the actual

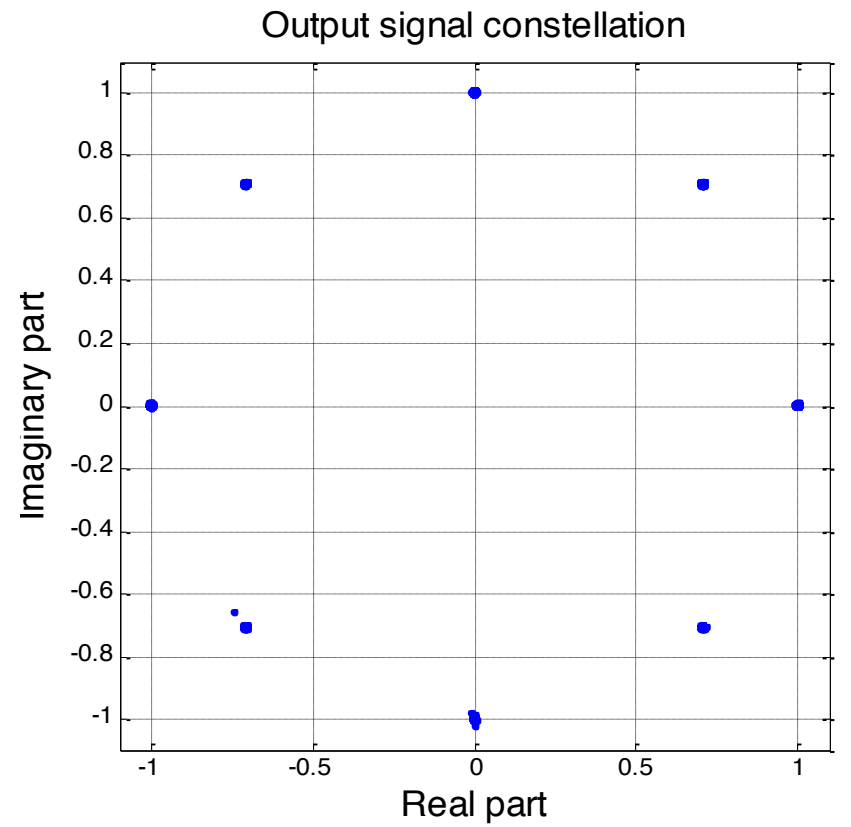

Fig. (6). On put constellations.

channel, ultimately affecting the equalizing effect of the equalizer. Therefore, adopting appropriate algorithm to reduce the mean square error and stabilize the error curve is our direction of future research.

\section{CONFLICT OF INTEREST}

The authors confirm that this article content has no conflict of interest.

\section{ACKNOWLEDGEMENTS}

The authors are grateful to the Heilongjiang province higher education teaching reform project in the new century 
and college students' science and technology innovation platform construction between China and Russia. The authors are also thankful to the Heilongjiang province higher education teaching reform project in the new century, colleges and universities of electronic design competition to promote teaching reform, research and practice.

\section{RERFERENCES}

[1] T. Ching-Hsiang, and E. J. Powers, "Nonlinear channel equalization in digital wireless systems," Global Telecommunications Conference, Houston, vol. 3, no. 11, pp. 1639-1643, 1993.

[2] S. Benedetto, E. Biglieri, and R. Daffara, "Modeling and performance evaluation of nonlinear wireless links-A volterra series approach“ Aerospace \& Electronic Systems Society, US, vol. 15, no. 4, pp. 494-507, 1979.
[3] T. Bouilloc, and G. Favier, "Nonlinear channel modeling and identification using baseband Volterra-Parafac models," Signal Processing, France, 2012, pp. 1492-1498.

[4] H. W. Kang, Y. S. Cho, and D. H. Youn, "An efficient adaptive predistorter for nonlinear high power amplifier in wireless communication," In: Circuits and Systems, Proceedings of 1997 IEEE International Symposium, British, 1997, vol. 4, no. 9-12, pp. 22882291.

[5] S. Jiang, "Nonlinear Channel Equalization Technology," Hangzhou University of Electronic Science and Technology, China, 2009.

[6] A. A. M. Saleh, "Frequency-independent and frequency-dependent nonlinear models of TWT amplifiers," IEEE Transactions on Communications, Canada, vol. 29, no. 11, pp. 1715-1720, 1981.

[7] F. Wang, K. Xing, and X. Xu, "Study on modeling method of nonlinear dynamic system," Computer Engineering and Application, China, vol. 45, no. 10, pp. 7-10, 2009.

(C) Min et al.; Licensee Bentham Open.

This is an open access article licensed under the terms of the Creative Commons Attribution Non-Commercial License (http://creativecommons.org/licenses/by-nc/4.0/) which permits unrestricted, non-commercial use, distribution and reproduction in any medium, provided the work is properly cited. 